\title{
GWAS-identified CCR1 and IL10 loci contribute to M1 macrophage-predominant inflammation in Behçet's disease
}

Hiroto Nakano ${ }^{1}$, Yohei Kirino ${ }^{{ }^{*}}$ (D), Mitsuhiro Takeno ${ }^{2}$, Kana Higashitani ${ }^{1}$, Hideto Nagai ${ }^{1}$, Ryusuke Yoshimi ${ }^{1}$, Yukie Yamaguchi ${ }^{3}$, Ikuma Kato ${ }^{4}$, Ichiro Aoki ${ }^{4}$ and Hideaki Nakajima ${ }^{1}$

\begin{abstract}
Background: Low C-C chemokine receptor 1 (CCR1) and interleukin (IL)-10 expression is associated with risk of Behçet's disease (BD). The objective of the present study was to clarify the pathological roles of CCRI and IL10 loci identified by previous BD genome-wide association studies (GWASs).

Methods: $M 1$ and $M 2$ macrophages $(M \varphi)$ were differentiated with granulocyte-macrophage colony-stimulating factor or macrophage colony-stimulating factor (M-CSF) from peripheral monocytes of healthy control subjects $(\mathrm{HC})$ and patients with BD. Expression of CD68 and CD163 was evaluated to test for M $\varphi$ polarization. CCR1 and IL-10 messenger RNA (mRNA) and protein expression was compared according to CCR1 and IL10 single-nucleotide polymorphism (SNP) genotypes. The migratory ability of M1 and M2 M 4 toward CCR1 ligand macrophage inflammatory protein (MIP)-1a was compared. The ratio of $\mathrm{M} 1$ and $M 2 M \varphi$ in skin lesions of BD and systemic sclerosis (SSc), which was reported to be M2 M 4 -dominant, was compared. To examine the plasticity of polarized $M \varphi$, the differentiated cells were cultured with either the same or the other culture condition.

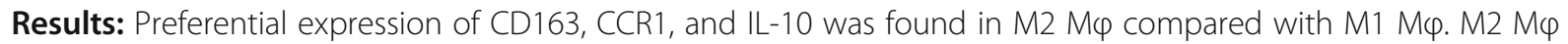

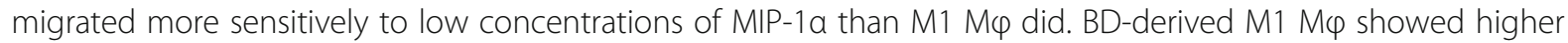
CCR1 surface expression than HC-derived M1 M $\varphi$ did. IL10 and CCR1 mRNA expression differences were observed

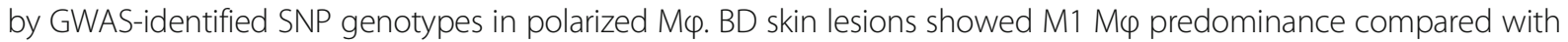
SSc skin lesions. A plasticity assay revealed that M-CSF restored IL-10 synthesis and reduced IL-6 production by M1 M $\varphi$.

Conclusions: The present study reveals that GWAS-identified SNPs contribute to $M 1 M \varphi$-predominant inflammation in $\mathrm{BD}$. Our data also suggest that the skewed $\mathrm{M} \varphi$ polarization is correctable by immunological intervention.
\end{abstract}

Keywords: Behçet's disease, Macrophage polarization, GWAS, CCR1, IL-10

\section{Background}

Behçet's disease (BD) is an idiopathic inflammatory disease that affects multiple organs, including the skin, eyes, vasculum, and mucosa [1, 2]. Genetic factors such as HLA-B $* 51$, as well as environmental factors, including microbial components, have been implicated in the pathogenesis of BD [3]. Recent genome-wide association studies (GWASs) followed

\footnotetext{
*Correspondence: kirino@yokohama-cu.ac.jp

${ }^{1}$ Yokohama City University Graduate School of Medicine, Department of Stem Cell and Immune Regulation, Yokohama, Japan

Full list of author information is available at the end of the article
}

by subsequent genetic studies have identified nearly 20 loci as the disease susceptibility genes, most of which are involved in both innate and acquired immune systems [4-10]. Of them, IL10 and CCR1 are highly expressed genes in macrophages $(M \phi)$. IL-10 is one of the cytokines conferring the anti-inflammatory effect, involved in the pathogenesis of various autoimmune diseases, including systemic lupus erythematosus (SLE) and inflammatory bowel diseases (IBD) [11]. Some studies have shown evidence that IL-10 is increased in BD inflamed tissues [12, 13]. However, genetic studies revealed that a BD risk single-nucleotide polymorphism (SNP) allele is 
associated with lower interleukin (IL)-10 production $[4,6]$, implicating complex interaction of T-helper cell Th1, Th2, and Th17 cytokines in BD [14].

CCR1 (C-C chemokine receptor 1), a membrane receptor for chemokines such as macrophage inflammatory protein (MIP)-1 $\alpha$, is known to be expressed on myeloid cells and is involved in cell migration [15]. We previously reported that the disease risk allele of IL1O locus rs1518111 is associated with reduced production of IL-10, as well as of CCR1 locus rs7616215, an SNP in the $3^{\prime}$ noncoding region, and that it is associated with lower CCR1 messenger RNA (mRNA) expression and reduced monocyte chemotaxis [5]. The result was unexpected because the low chemotactic ability of monocytes, which seems to be protective from inflammation, was identified as a risk for BD. This genetic findings do not directly correspond to previous functional studies that have shown excessive production of $\mathrm{M} \phi$-derived proinflammatory cytokines, including tumor necrosis factor (TNF)- $\alpha$, which is the most effective therapeutic target in $\mathrm{BD}[16,17]$. Additionally, our recent genetic study suggested that an effective target may be the IL1A-IL1B locus, which is related to enhanced IL-1 $\beta$ production from activated $\mathrm{M} \phi$ [10].

$\mathrm{M} \phi$ polarization concepts have gained attention, especially in the field of tumor immunity [18]. Further studies have revealed that $M \phi$ are divided into two major subtypes with distinct functions: $\mathrm{M} 1$ and $\mathrm{M} 2 \mathrm{M} \phi$. M1 $\mathrm{M} \phi$ are classical $M \phi$ and play a proinflammatory role through the production of IL- 6 and TNF- $\alpha$, and they activate the Th1 cellular immune response, whereas $\mathrm{M} 2 \mathrm{M} \phi$ are nonclassical $\mathrm{M} \phi$ which produce antiinflammatory cytokine IL-10 that suppresses inflammatory responses [19]. The functional and phenotypic diversity of $M \phi$ has been also investigated in physiological and other pathological conditions [20]. Recent GWASs have identified IL1O and related genes as susceptibility genes associated with immune-mediated diseases such as IBD and SLE in addition to BD, suggesting the involvement of abnormal M2 M $\phi$ function in the pathogenesis of these diseases [21]. Indeed, dysfunction of M2 M $\phi$ has been shown to exacerbate inflammation in a herpes simplex virus-induced BD mouse model [22]. We previously revealed that downregulation of heme oxygenase (HO)-1, which is preferentially expressed as an $\mathrm{M} 2 \mathrm{M} \phi$-specific anti-inflammatory protein, suggested M2 M $\phi$ dysfunction in BD [23]. These findings prompted us to analyze functional studies of $\mathrm{M} \phi$ polarization in human patients with BD. The results of the present study support the hypothesis that GWASidentified CCR1 and IL1O loci contribute to impaired $\mathrm{M} 2 \mathrm{M} \phi$ function, which is partly due to genetic predisposition, and lead to M1 M $\phi$-predominant immune responses in $\mathrm{BD}$.

\section{Methods}

\section{Study subjects}

All of the individuals with BD in this study fulfilled the International Study Group (ISG) criteria for BD [24]. The study protocol and genetic analyses were approved by the Yokohama City University Hospital Ethics Board (A140327015 and B131107009). All of the human samples were used after written informed consent was obtained.

\section{Cell preparation and culture}

Peripheral blood mononuclear cells (PBMCs) were isolated by density gradient centrifugation using Lymphoprep (Axis-Shield, Dundee, UK). Human monocytes were isolated from PBMCs using the Human Monocyte Isolation Kit II magnetic-activated cell sorter (Miltenyi Biotec, Bergisch-Gladbach, Germany) according to the manufacturer's protocol [23]. Isolated monocytes were plated at a density of $5 \times 10^{5}$ cells $/ \mathrm{ml}$ and were incubated in RPMI 1640 medium (R8758; SigmaAldrich, St. Louis, MO, USA) supplemented with $10 \%$ FBS (MP Biomedicals, Santa Ana, CA, USA) in the presence of either recombinant $20 \mathrm{ng} / \mathrm{ml}$ human granulocyte-macrophage colony-stimulating factor (GMCSF) (215-GM; R\&D Systems, Minneapolis, MN, USA) or $50 \mathrm{ng} / \mathrm{ml}$ macrophage colony-stimulating factor (MCSF) (216-MC; R\&D Systems) for 9 days to induce differentiation to M1 or M2 M $\phi$, respectively [25].

\section{Flow cytometry}

Antibodies for CD68 (FITC 333806), CD163 (PE-Cy7 333614), and CCR1 (PE 362904) were purchased from BioLegend (San Diego, CA, USA). Incubation of polarized $M \phi$ with antibodies was performed according to the manufacturer's protocol. M1 and M2 M $\phi$ were defined as $\mathrm{CD} 68^{+} \mathrm{CD} 163^{-}$and $\mathrm{CD} 68^{+} \mathrm{CD} 163^{+}$cells, respectively. All the gates were determined using fluorescence minus one control. Data were collected on a BD FACSCanto II instrument (BD Biosciences, San Jose, CA, USA) and analyzed using FlowJo software version 10 (FlowJo, Ashland, OR, USA).

\section{IHC (Immunochemical) staining}

Cultured cells were fixed on Nunc Lab-Tek chamber slides (Thermo Fisher Scientific, Waltham, MA, USA) with $4 \%$ paraformaldehyde. The slides and paraffinized skin specimens obtained from four patients with BD and four patients with systemic sclerosis (SSc) were deparaffinized in xylene, hydrated in ethanol, and pretreated with $10 \mathrm{mmol} / \mathrm{L}$ citrate buffer ( $\mathrm{pH}$ 6.0). The cells and tissues were used for IHC analysis. Mouse monoclonal anti-CD68 antibody (PGM1; Dako, Carpinteria, CA, USA), anti-CD163 antibody (Leica Biosystems, Berlin, Germany), and rabbit polyclonal immunoglobulin G (IgG) anti-CCR1 antibody (GenWay Biotech, San Diego, 
CA, USA) at 1:100 dilution were used as primary antibodies. The REAL EnVision Detection System (Dako) was used with secondary antibodies for visualization by light microscopy [26]. To count M1 and M2 Mф in skin lesions, $\mathrm{CD}^{+} 8^{+}$and $\mathrm{CD}_{163^{+}}$cells were counted in three high-power fields (magnification $\times 400$ ), and the average CD68/CD163 ratio was obtained in each patient. For double-immunofluorescence staining, Alexa Fluor 555 anti-mouse IgG (Life Technologies, Carlsbad, CA, USA) and Alexa Fluor 488 anti-rabbit IgG (Life Technologies) at 1:500 dilution were used as secondary antibodies. The slides were mounted with 4',6-diamidino-2-phenylindole (Vector Laboratories, Peterborough, UK) and imaged by fluorescence microscopy.

\section{Semiquantitative mRNA analysis}

mRNA was extracted using the RNeasy kit (Qiagen, Hilden, Germany), and complementary DNA (cDNA) was synthesized using the SuperScript III reverse transcriptase (Life Technologies). Primers for real-time PCR of CD163 (Hs00174705_m1), CCR1 (Hs00174298_m1), CCR2 (Hs00704702_s1), IL6 (Hs00174131_m1), and IL10 (Hs00961622_m1) were purchased from Applied Biosystems (Foster City, CA, USA). GAPDH (4310884E) was used as an internal control when performing multiplex PCR. The comparative cycle threshold $\left(2^{-\Delta \Delta \mathrm{Ct}}\right)$ method was used for the analysis of duplicate or triplicate reactions [4].

\section{Measurements of cytokines}

Lipopolysaccharide (LPS) from Escherichia coli (serotype 0111:B4; InvivoGen, San Diego, CA, USA) was used at $1 \mu \mathrm{g} / \mathrm{ml}$. After incubation for 24 hours, the supernatants were centrifuged and stocked at $-80{ }^{\circ} \mathrm{C}$ until use. The concentrations of TNF- $\alpha$, IL- 6 , and IL-10 were quantified using the BD Bead Assay (BD Biosciences) in accordance with the manufacturer's protocol.

\section{Migration assay}

Transwell migration assay was performed using $5-\mu \mathrm{m}$ pore polycarbonate membrane (Corning Costar, Kennebunk, ME, USA). M1 and M2 M $\phi$ were cultured on RepCell (CellSeed, Tokyo, Japan) [27]. After detachment of cells incubated at $20{ }^{\circ} \mathrm{C}$ for 30 minutes, the cells were used for the following experiments. M1 or M2 M $\phi\left(5 \times 10^{4}\right)$ were applied in the upper chamber. In the lower chamber, $1 \%$ bovine serum albumin (Wako Pure Chemical Co., Osaka, Japan)-supplemented RPMI 1640 medium with or without recombinant human MIP-1 $\alpha$ (R\&D Systems) was applied. After incubation for 2 hours, cells in the upper chamber were removed with Q-Tips, and cells on the bottom side of the membrane were stained with Diff-Quik (Sysmex, Kobe, Japan). Cells were randomly counted in 25 high-power fields. The migration index was defined as the ratio between the migrated cell counts with or without MIP- $1 \alpha$.

\section{Plasticity assay}

After a 9-day incubation of monocytes from BD or SSc with GM-CSF or M-CSF, polarized M1 or M2 M $\phi$ were further incubated in the presence of GM-CSF or M-CSF for another 9 days (Fig. 4a). cDNA was obtained after the 18-day incubation.

\section{SNP genotyping}

Genomic DNA was isolated with the DNeasy Blood \& Tissue Kit (Qiagen) or BuccalQuick (Trimgen, Sparks, MD, USA). A TaqMan SNP genotyping assay was used to identify SNPs associated with expression of IL10 (C__8828803_1_; Applied Biosystems) and CCR1 (C

1198110_10; Applied Biosystems) [4].

\section{Statistical analysis}

Student's unpaired $t$ test was used to test statistical significance. A $P$ value of $<0.05$ was considered significant. One-way analysis of variance was performed to compare three groups, except for the groups that did not contain sufficient samples suitable for analysis owing to allelic scarcity. In the latter instance, we combined heterozygous with homozygous minor alleles and analyzed them using an unpaired Student's $t$ test. Statistical analysis was performed using Prism software (GraphPad Software, La Jolla, CA, USA).

\section{Results}

$\mathrm{M} \varphi$ polarization in human circulating monocytes

First, we tested the $\mathrm{M} \phi$ polarization protocol in peripheral monocytes purified from healthy control subjects (HC) according to a description in a previous report [25]. M1 and $\mathrm{M} 2 \mathrm{M} \phi$ differentiation was induced from peripheral monocytes by GM-CSF and M-CSF, respectively. The cultured cells showed distinct morphological features between the two culture systems (Additional file 1: Figure S1A and B). We confirmed that both mRNA and CD163 protein, one of the conventional M2 markers, were elevated in M2- rather than M1-cultured cells (Additional file 1: Figure S1C and D) [28]. Cytokine profiles after 24-h LPS stimulation revealed that M1-cultured cells produced a higher amount of IL-6, whereas M2- but not M1-cultured cells secreted IL-10 (Additional file 1: Figure S1E and F). Both types of cells did not synthesize a detectable level of either cytokine in the absence of LPS. These data indicate that both phenotypic features and cytokine profiles of cells derived from M1 and M2 conditions were compatible with those of M1 and M2 M $\phi$. Thus, we applied this protocol in the following experiments. 


\section{CCR1 and IL-10 expression by M1 and M2 M $\varphi$ differentiated in vitro}

Having established the $\mathrm{M} \phi$ polarization protocol, we compared CCR1 expression and IL-10 production, both of which are identified as BD susceptible genes, by M1 and M2 M $\phi$. We found that M2 M $\phi$ showed higher amounts of CCR1 mRNA and protein than M1 M $\phi$ (Fig. 1a and b). Consistently, immunocytochemical analysis confirmed that CD163 and CCR1 were more abundant in M2 M $\phi$ than in $\mathrm{M} 1 \mathrm{M} \phi$ (Fig. 1c).

We next looked at the association between IL10 and CCR1 mRNA expression and their corresponding BDassociated SNP genotypes. We found that BD risk allele was associated with decreased expression of both CCR1 and IL10 mRNA production in polarized M $\phi$. A significant expression quantitative trait loci (eQTL) effect was found only in M1 M $\phi$ for CCR1 and IL10 for M2 M $\phi$ (Fig. 1d and e). A recent study demonstrated that rs7616215 has a robust eQTL effect on CCR2, but not CCR1, in monocytes [29]. However, the result was not replicated in our polarized $\mathrm{M} \phi$ assay (Additional file 1: Figure S2A).

To examine whether the upregulated CCR1 expression is associated with enhanced chemotaxis against CCR1 ligands, we used a Transwell migration assay with gradient density of MIP-1 $\alpha$, a ligand for CCR1. We found that the migration of $\mathrm{M} 1 \mathrm{M} \phi$ into the lower chamber occurred in relatively higher MIP- $1 \alpha$ concentrations than M2 M $\phi$ (Fig. 1f). However, the migration of M2 $\mathrm{M} \phi$ into the lower chamber was more efficient at low concentrations of MIP- $1 \alpha$ than M1 M $\phi$, whereas the chemotaxis was rather inhibited with higher concentrations of the chemokine (Additional file 1: Figure S3). Similar findings were reported previously in an unrelated study using this assay system [30]. These findings indicate that increased CCR1 expression contributes to sensitive chemotaxis in response to low levels of MIP- $1 \alpha$ in M2 M $\phi$. These results support the notion that BDassociated CCR1 and IL1O loci have the capacity to alter migration and anti-inflammatory cytokine production in polarized $\mathrm{M} \phi$.

\section{$M 1$ and $M 2 M \varphi$ in patients with $B D$}

To ask whether the CCR1 expression is affected by the disease itself, we compared the frequency of CCR1positive M1 and M2 M $\phi$ from $\mathrm{HC}$ and BD. To carry out this experiment, 21 patients with BD who fulfilled ISG criteria were enrolled. The background characteristics of the patients and $\mathrm{HC}$ are shown in Additional file 1: Table S1 and Table S2, respectively. The results showed that CCR1 surface expression of M1 M $\phi$ was significantly increased in BD compared with $\mathrm{HC}$ (Fig. 2a), whereas no difference was detected in M2 M $\phi$ (Fig. 2b). No significant difference between $\mathrm{BD}$ and $\mathrm{HC}$ was observed in CCR1 mRNA expression. The eQTL effects of rs7616215 on CCR1/2 mRNA and protein are shown
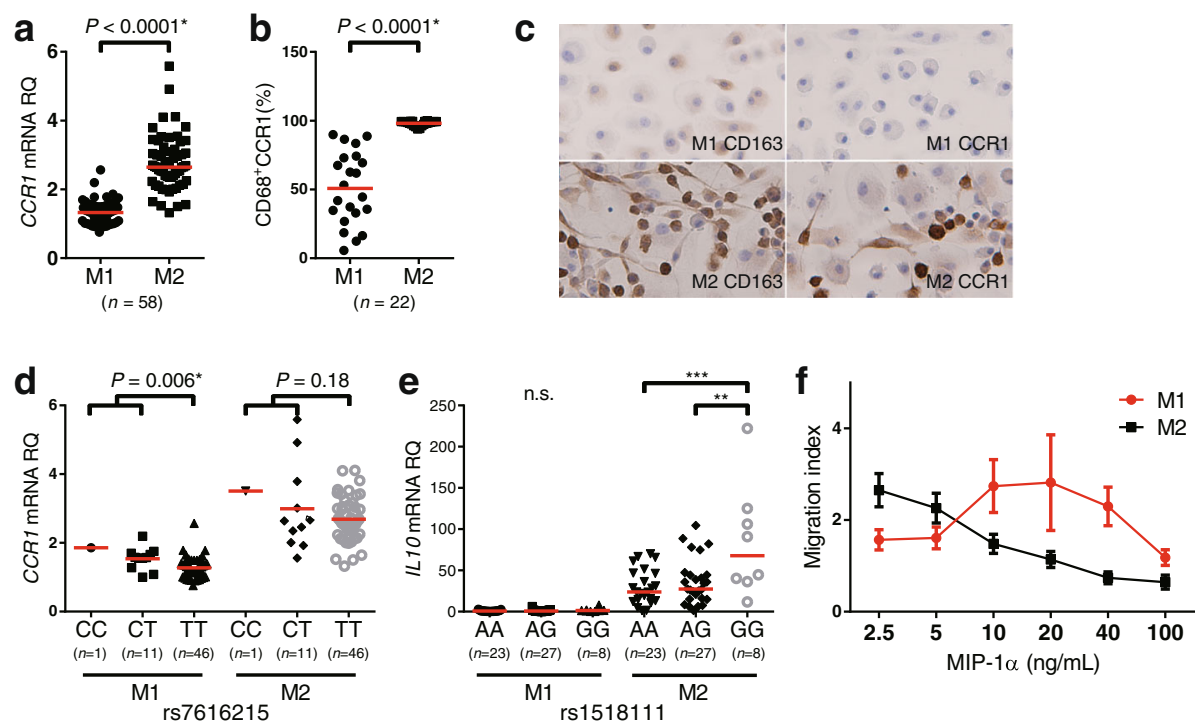

Fig. 1 Comparison of M2 marker expression and function between $M 1$ and $M 2$ macrophages (M $\varphi$ ) derived from healthy control subjects (HC). C-C chemokine receptor 1 (CCR1) messenger RNA (mRNA) and surface protein expression of M1 and M2 M $\varphi$ were detected using (a) real-time

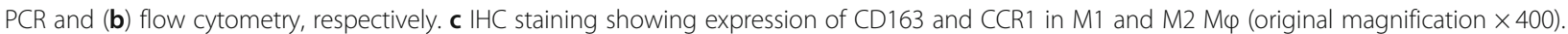
d CCR1 single-nucleotide polymorphism (SNP) rs7616215 genotype and expression of CCR1 mRNA and (e) IL10 SNP rs1518111 genotype and IL10 mRNA expression in M1 and M2 M $\varphi$. f Chemotaxis of $\mathrm{M} 1$ (red) and $\mathrm{M} 2 \mathrm{M} \varphi$ (black) toward macrophage inflammatory protein (MIP)-1a were determined by Transwell migration assay. The results are shown as the migration index. Red horizontal bars indicate median values. $P$ values were determined by Student $t$ test and one-way analysis of variance $\left(* * P<0.01,{ }^{* * *} P<0.001\right)$. RQ Relative quantity 

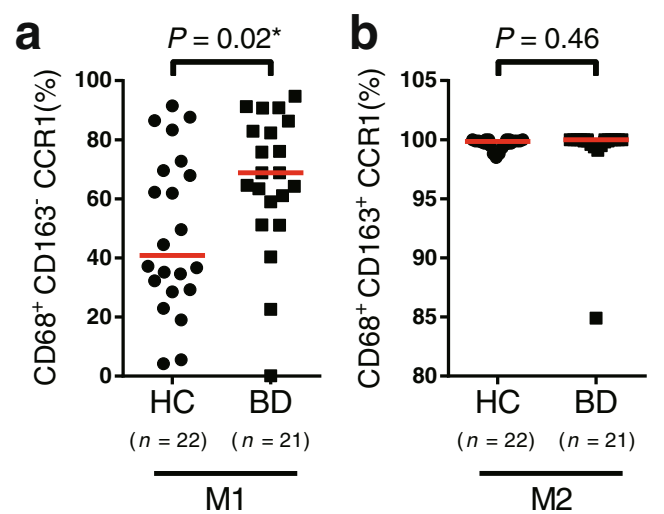

M2

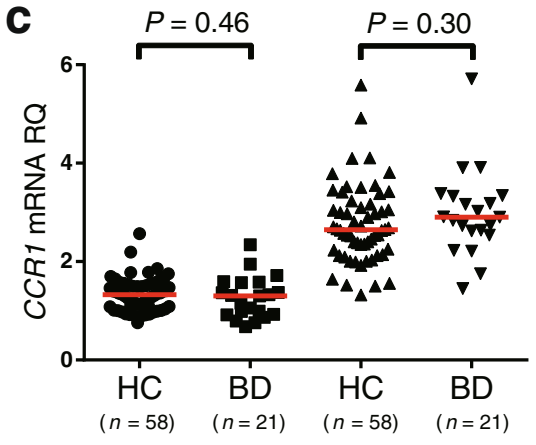

M1
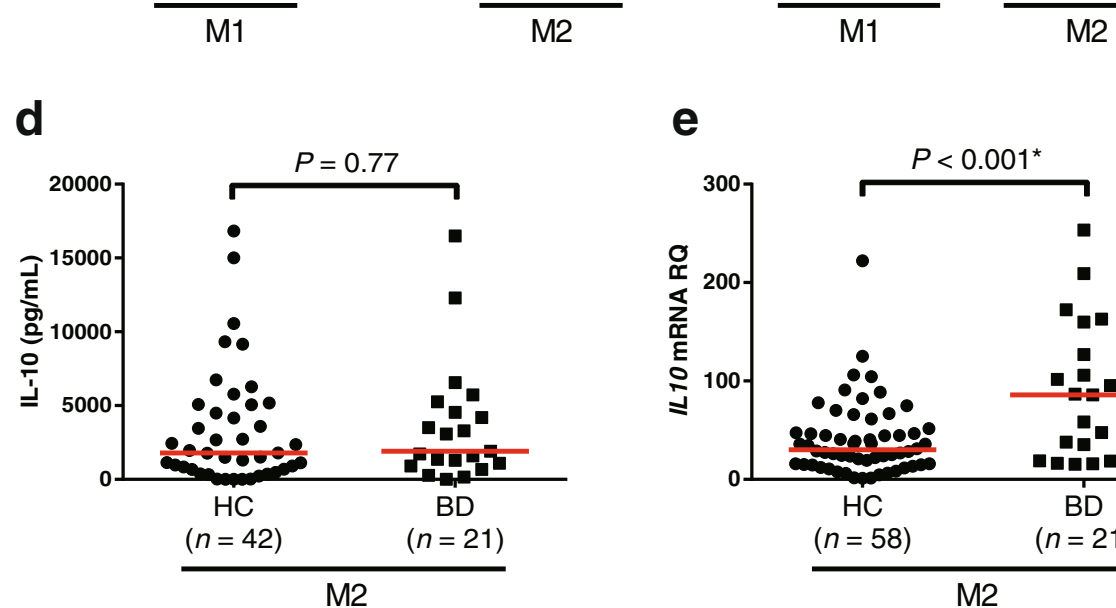

$\mathbf{e}$

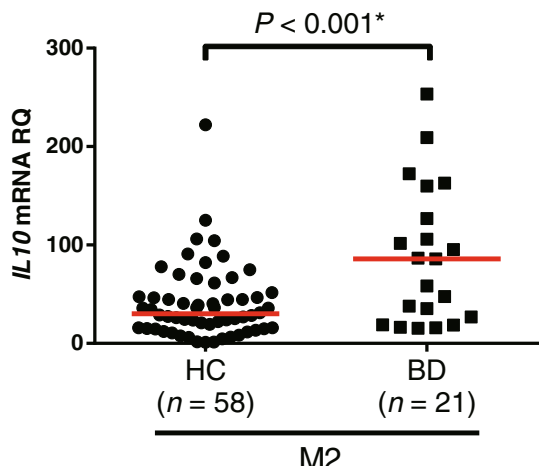

Fig. 2 Comparison of C-C chemokine receptor 1 (CCR1) and interleukin (IL)-10 expression in macrophages (M $\varphi$ ) derived from between healthy control subjects $(\mathrm{HC})$ and patients with Behçet's disease (BD). Flow cytometric analysis of $\mathrm{CD}^{+} 8^{+} \mathrm{CD} 163^{-} \mathrm{CCR} 1^{+}(\mathrm{M} 1 \mathrm{M} \varphi)$ cells $(\mathbf{a})$ and

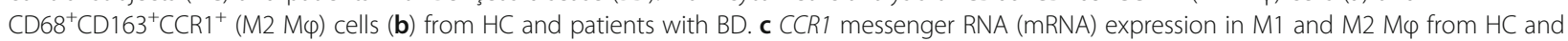
patients with BD. d Supernatant IL-10 concentration of lipopolysaccharide-stimulated M $\varphi$ from HC and patients with BD. e IL10 mRNA expression in $\mathrm{M} 1$ and $\mathrm{M} 2 \mathrm{M} \varphi$ from $\mathrm{HC}$ and patients with $\mathrm{BD}$. Red horizontal bars indicate median values. $P$ values were determined by Student's $t$ test. $R Q$ Relative quantity

in Additional file 1: Figure S2. The statistically significant allele-specific expression was seen only in HC.

We confirmed that no statistically significant difference in CCR1 positivity was found between treatment with or without biologics, colchicine, or prednisolone (Additional file 1: Figure S4). We also investigated the number of differentiated $\mathrm{M} \phi$ between $\mathrm{HC}$ and $\mathrm{BD}$, although no significant difference was observed between the groups (Additional file 1: Figure S5).

IL-10 protein and mRNA expression in $\mathrm{M} 2 \mathrm{M} \phi$ from $\mathrm{HC}$ and BD is shown in Fig. $2 \mathrm{~d}$ and e. IL10 mRNA expression was elevated in $\mathrm{BD}$, whereas IL-10 protein production was comparable between $\mathrm{BD}$ and $\mathrm{HC}$. The eQTL effect of rs1518111 on IL-10 protein and mRNA is shown in Additional file 1: Figure S6. Statistically significant allelespecific expression was seen only in HC IL10 mRNA.

\section{$M 1$ and $M 2 M \varphi$ in skin lesions of BD and systemic} sclerosis

GWASs and our in vitro data suggested that GWASidentified CCR1 SNPs confer significant eQTL effects in polarized $M 1 M \phi$, which may lead to skewed $M \phi$ polarization balance in BD local inflammation. To prove this concept, we performed IHC staining of erythema nodosum, which was pathologically diagnosed as septal panniculitis, from the lower limbs of a treatment-naïve patient with $\mathrm{BD}$. We used skin lesions of SSc as a control, which was recently reported to be M2-dominant [31].

As shown in Fig. 3a, CD68 ${ }^{+}$cells are predominant over $\mathrm{CD}_{163}{ }^{+}$cells in skin lesions of patients with BD compared with patients with SSc (Fig. 3b). CCR1 and CD163 double-positive cells were found in the skin lesions of patients with BD (Fig. 3c). These results suggest M1 $\mathrm{M} \phi$-dominant inflammation in BD.

\section{Plasticity in human M1 and M2 M $\varphi$}

As shown in Additional file 1: Figure S1F, M2 M $\phi$ have the capacity to produce IL-10, which has potent antiinflammatory effects. Lower IL-10 production is a risk for not only BD but also ulcerative colitis [32] and other inflammatory diseases. IL10R-deficient patients experience 


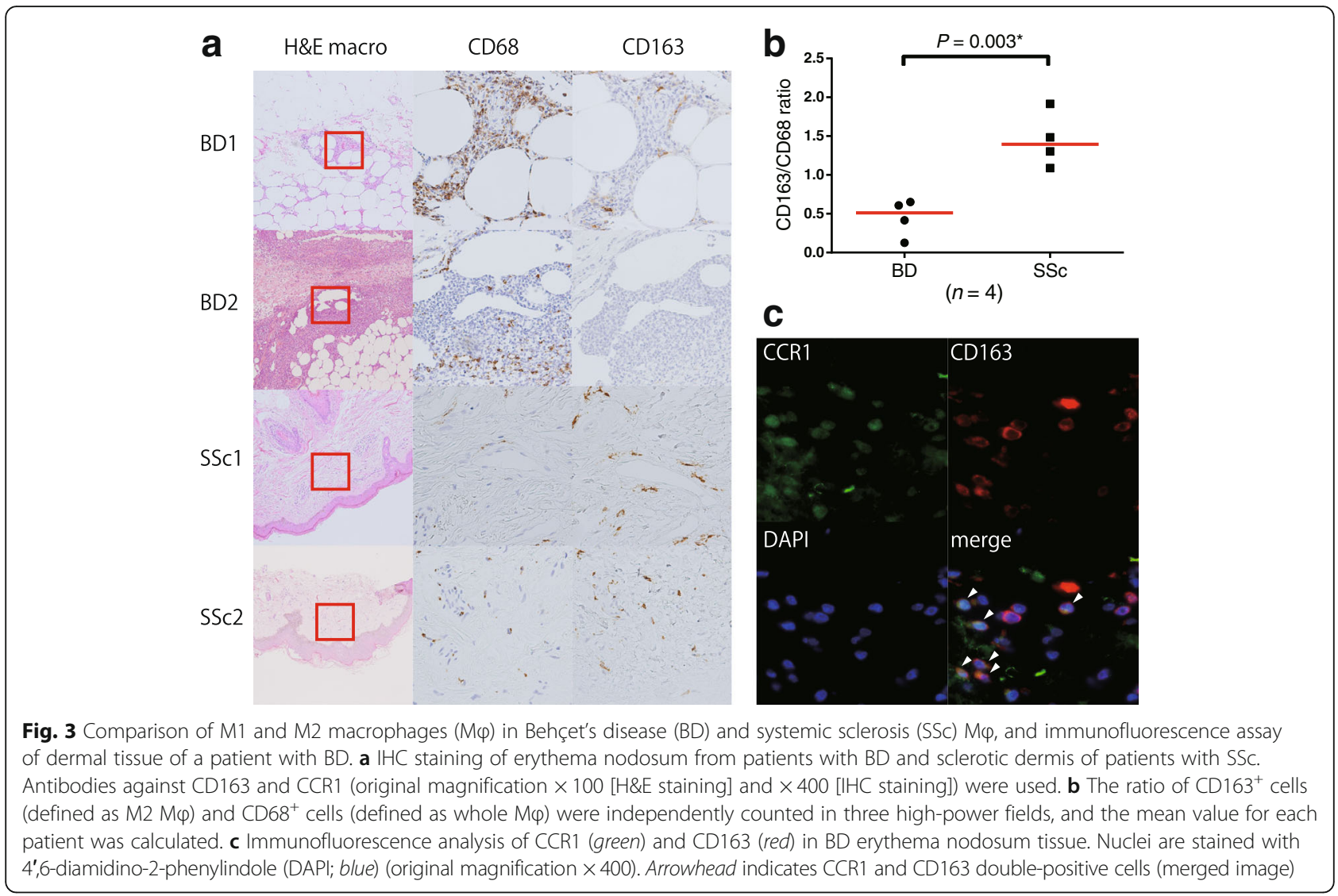

oral ulceration and IBD, phenotypes resembling BD [33]. It would be of great benefit for patients with $\mathrm{BD}$ and other inflammatory diseases if IL-10 supplementation restored $\mathrm{M} 2 \mathrm{M} \phi$ function at inflammatory sites, leading to a favorable clinical outcome.

We tested whether established M1 M $\phi$ could be converted into $\mathrm{M} 2 \mathrm{M} \phi$ in humans. To this end, the M1 and $\mathrm{M} 2 \mathrm{M} \phi$ that underwent 9-day in vitro polarization by GM-CSF or M-CSF were cultured for an additional 9 days in the presence of the same or other cytokines (Fig. 4a). Expression of CD163, IL6, IL10, and CCR1 mRNA was measured at the end of culture (Fig. 4b-e). We found that treatment with M-CSF, but not with GM-CSF, restored expression of IL10 mRNA with downregulation of IL6 mRNA. On the contrary, IL10 mRNA expression was decreased by GM-CSF in M2 M $\phi$. Thus, the final expression patterns of CD163, IL6, IL10, and CCR1 mRNA depended on the second cytokine rather than on the first one. The results suggest that $M \phi$ phenotypes are partly but not completely interchangeable between $\mathrm{M} 1$ and $\mathrm{M} 2 \mathrm{M} \phi$, depending on circumstantial factors, including cytokines.

\section{Discussion}

In the previous GWASs and subsequent genetic studies, associations of $I L 10[4,6,14]$ and CCR1 [5, 34] loci with
BD were independently replicated in multiple populations. Therefore, they are now considered bona fide loci involved in the pathogenesis of $\mathrm{BD}$ [35], though the pathological role remains uncertain regarding the functional basis.

The present study shows that both ILIO and CCRI genes were preferentially expressed on $\mathrm{M} 2 \mathrm{M} \phi$, suggesting that the disease-susceptible SNPs of the genes are responsible for impaired migration of $\mathrm{M} 2 \mathrm{M} \phi$ into the inflammatory lesions and reduced local synthesis of IL10 in BD. Thus, although IL10 and CCR1 SNPs are independently identified as susceptible loci of $\mathrm{BD}$, it is likely that their pathogenic roles are summarized into the impaired $\mathrm{M} 2 \mathrm{M} \phi$ function in $\mathrm{BD}$.

Anti-inflammatory effects of IL-10 are mediated by HO-1, which was previously shown to be expressed on M2 M $\phi$ preferentially [36, 37]. We have previously shown defective HO-1 synthesis to be associated with excessive expression of Toll-like receptor 4, another disease-susceptible gene product, leading to amplification of inflammation in patients with BD [23, 38]. These findings provided additional circumstantial evidence of impaired $\mathrm{M} 2 \mathrm{M} \phi$ functions in $\mathrm{BD}$.

Although biologics targeting IL-10 would be useful for the treatment of inflammatory diseases, there are no commercially available drugs that directly manipulate IL-10 levels in humans. In animals, enhancing M2 M $\phi$ 

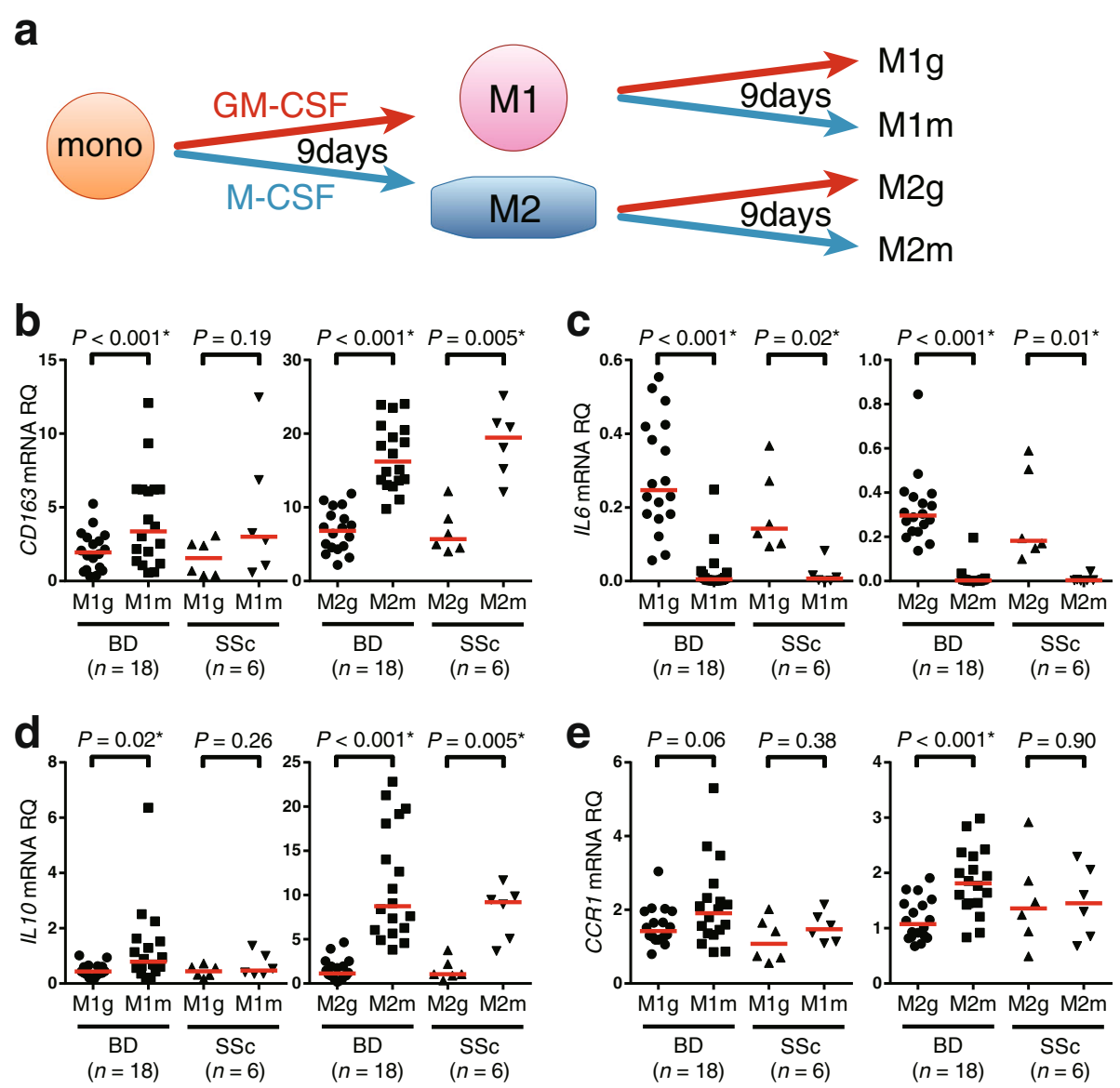

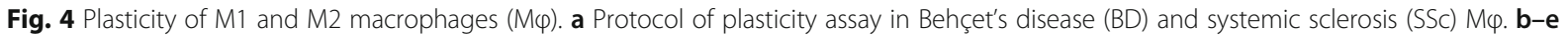
CD163 (b), IL-6 (c), IL-10 (d), and CCR1 (e) mRNA expression from M1g, M1m, M2g, and M2m cells are shown. Red horizontal bars indicate median values. GM-CSF Granulocyte-macrophage colony-stimulating factor, M-CSF Macrophage colony-stimulating factor, RQ Relative quantity

has been shown to be protective in several SLE models $[39,40]$. It is plausible that a treatment strategy could be directed at enhancement of IL-10 production or local accumulation of $\mathrm{M} 2 \mathrm{M} \phi$ into inflammatory lesions, contributing to the improvement of clinical outcomes of BD. TNF inhibitors may lead to a relative enrichment of M2 $M \phi$ by inhibiting M1 M $\phi$ function, because we have previously shown that HO-1 mRNA expression in circulating monocytes is increased during treatment with infliximab in patients with rheumatoid arthritis [41]. Alternatively, apremilast, a phosphodiesterase 4-selective inhibitor for which an international clinical trial for BD is ongoing, stimulates the production of IL-10 and downregulates that of proinflammatory cytokines [42]. These findings suggest that clinical efficacy of these treatments is associated with corrected M1/M2 balance for BD, though direct evidence has not been provided to date.

A significant difference between $\mathrm{BD}$ and $\mathrm{HC}$ was observed in CCR1 protein expression but not in CCR1 mRNA expression. We could not detect genotype-dependent IL-10 expression differences in BD. Rather, IL10 mRNA expression was higher in patients with BD (Fig. 2e). We posit the following possibilities:

1. Because the disease-protective GG genotype of IL10 SNP rs1518111 and the CC genotype of CCR1 SNP rs7616215 in patients with $\mathrm{BD}$ were small, the power to identify mRNA expression differences was limited in BD samples.

2. Effects of disease status or treatments could have affected IL-10 expression.

3. The sample size was too small to identify small expression differences caused by the SNP.

However, because SNPs tag common DNA haplotypes in human populations, data identified in healthy individuals would be applicable in BD pathogenesis. Further IL10 and CCR1 transcriptional analysis is needed to clarify whether IL-10 and CCR1 expression may be dysregulated in $\mathrm{BD}$. 
There has been a discrepancy regarding the eQTL effect of rs7616215 genotype on CCR1/2 expression between this report and a former one [29]. We speculate the following possibilities:

1. The examined cells are different; we evaluated M1 and M2 macrophages, whereas Ishigaki et al. evaluated human peripheral monocytes.

2. The assay used to detect mRNA expression was different; we used real-time PCR, whereas Ishigaki et al. used RNA sequencing.

3. The sample size is too small to identify CCR2 expression differences in our study.

However, in our previous paper [5], we found a similar CCR1 eQTL pattern in human peripheral monocytes in samples from white Americans as well as in publicly available mRNA array data [43]. These data support our hypothesis that rs7616215 affects CCR1 mRNA expression.

We could not evaluate CCR1 genotype dependent migration differences in the present study for the following reasons: (1) 50-ml blood samples were needed to obtain a large-enough M1 or M2 macrophage count for the Transwell migration assay, and (2) owing to large experimental variation of the Transwell migration assay of polarized macrophages, a large sample size would be necessary to detect subtle effects caused by the common SNP. However, as we have previously shown significant migration differences by rs7616215 genotypes in human monocytes [5], similar migration differences may be found in human macrophages as well.

Figure 5 shows our hypothesis based on the present study. M2 M $\phi$ expressing high levels of CCR1 have the advantage of migration to inflammatory lesions, where CCR1 ligands such as MIP- $1 \alpha$ are secreted, compared with M1 M $\phi$. Subsequently, the local inflammation is decreased by $\mathrm{M} 2 \mathrm{M} \phi$ in healthy individuals. However, the inflammatory condition would be persistent in patients with BD because $M 2 M \phi$ function is impaired by the genetic predisposition. Local accumulation of M2 $\mathrm{M} \phi$ is decreased owing to the reduced CCR1-dependent chemotaxis. Local IL-10 production would also be much less in patients with $\mathrm{BD}$ than in $\mathrm{HC}$ owing to the reduced number of $\mathrm{M} 2 \mathrm{M} \phi$ in addition to low IL-10synthesizing capacity. Furthermore, the frequency of CCR1-positive $\mathrm{M} 1 \mathrm{M} \phi$ is rather increased in patients with $\mathrm{BD}$ compared with $\mathrm{HC}$, suggesting a more efficient accumulation of M1 M $\phi$ into MIP- $1 \alpha$-enriched inflammatory lesions in $\mathrm{BD}$ than in $\mathrm{HC}$. These unexpected findings may be caused by epigenetic or environmental factors [44]. Taken together, both quantitative and functional balance between $\mathrm{M} 1$ and $\mathrm{M} 2 \mathrm{M} \phi$ shift toward

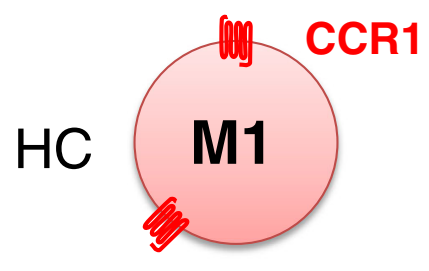

Phenotype dependent CCR1 expression $\uparrow$ BD

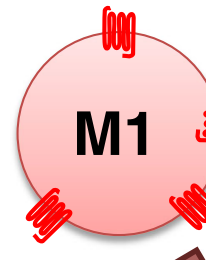

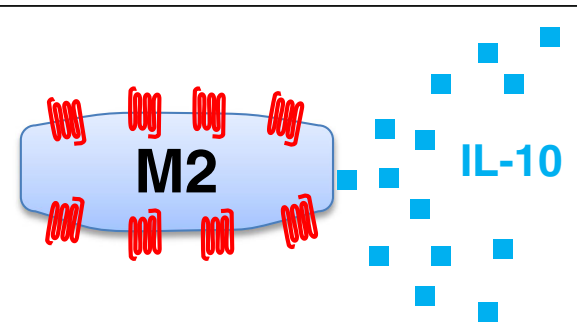

Genotype dependent CCR1 expression $\downarrow$ (rs7616215)

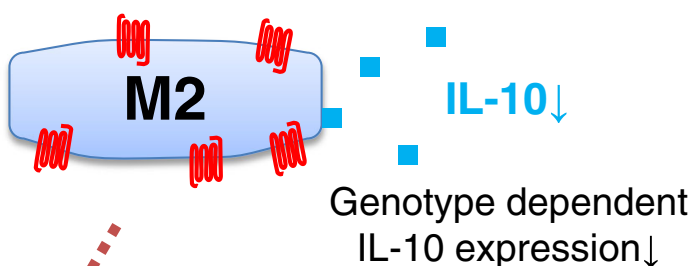

(rs1518111)

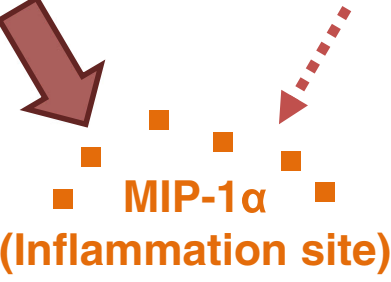

Fig. 5 The hypothesis of C-C chemokine receptor 1 (CCR1) and interleukin (IL)-10 function in Behçet's disease (BD) macrophages. MIP-1a Macrophage inflammatory protein, HC Healthy control subjects 
M1-predominant type in inflammatory lesions, resulting in a vicious cycle of local inflammation. We added our new hypothesis to the previously proposed schema representing the pathogenesis of $\mathrm{BD}$ (Additional file 1: Figure S7) [45, 46].

Phenotypic plasticity of matured $M \phi$ is an important issue for future study. Our data indicated that $M \phi$ function was modified by M-CSF or GM-CSF at least in vitro. Particularly, we confirmed that in vitro polarized M1 M $\phi$ restored IL-10 synthesis capacity in the presence of MCSF. Even such partial modification of $\mathrm{M} \phi$ function would be beneficial for patients with $\mathrm{BD}$.

This study has some limitations. First, most of the data were obtained from in vitro experiments, which might not fully reflect the in vivo biological events in patients with BD. Second, because biopsies of affected lesions in $\mathrm{BD}$ are not routinely done for the diagnosis of $\mathrm{BD}$, only small numbers of skin biopsy specimens could be evaluated. In addition, the timing of skin biopsy in the disease course may influence the balance between M1 and M2 $\mathrm{M} \phi$. Finally, most of the patients with BD were receiving some of the therapeutic agents, which might have affected the results of in vitro culturing. However, the 9day in vitro polarization protocol may have minimized the influence of the therapeutic agents, as shown in Additional file 1: Figure S4. Nonetheless, our data reveal the difference in CCR1 expression of $\mathrm{M} 1 \mathrm{M} \phi$ between patients with $\mathrm{BD}$ and $\mathrm{HC}$.

\section{Conclusions}

The present study shows that $\mathrm{BD}$-associated $C C R 1$ and IL10 loci are responsible for defective $\mathrm{M} 2 \mathrm{M} \phi$, resulting in skewed M1 M $\phi$ polarization in BD. Our data suggest that phenotypic plasticity is partially conserved in matured M1 and M2 M $\phi$. Strategies to correct the imbalance between $M 1$ and $M 2 M \phi$ or to modify $M \phi$ function would be promising for patients with $\mathrm{BD}$ in the future.

\section{Additional file}

Additional file 1: Figure S1. Comparison of phenotypic features and cytokine profiles between $\mathrm{M} 1$ and $\mathrm{M} 2$ cultured cells. Figure S2. eQTL effect of rs7616215 on CCR2 and CCR1. Figure S3. Detailed results of chemotaxis of $\mathrm{M} 2 \mathrm{M} \varphi$ toward various concentrations of MIP-1a. Figure S4. Differences in CCR1 positivity (fluorescence-activated cell sorting) between treatments. a Biologics. b Colchicine. c Prednisolone. Figure S5. The number of differentiated $M \varphi$ in vitro in $\mathrm{HC}$ and $\mathrm{BD}$. Figure S6. eQTL effect of rs1518111 on IL-10 protein and mRNA. Figure S7. Schema showing our proposed immunological responses in BD. Table S1. Characteristics of patients with BD who participated in the study. Table S2. Characteristics of HC study participants. (DOCX $9663 \mathrm{~kb}$ )

\section{Abbreviations}

BD: Behçet's disease: CCR: C-C chemokine receptor; CD: Cluster of differentiation; CDNA: Complementary DNA; DAPI: 4',6-Diamidino-2phenylindole; eQTL: Expression quantitative trait loci; GAPDH: Glyceraldehyde 3-phosphate dehydrogenase; GM-CSF: Granulocyte-macrophage colony- stimulating factor; GWAS: Genome-wide association study; HC: Healthy control subjects; HO: Heme oxygenase; IBD: Inflammatory bowel disease; IgG: Immunoglobulin G; IL: Interleukin; ISG: International Study Group; LPS: Lipopolysaccharide; M-CSF: Macrophage colony-stimulating factor; MIP: Macrophage inflammatory protein; mRNA: Messenger RNA; M $\varphi$ : Macrophages; PBMC: Peripheral blood mononuclear cells; SLE: Systemic lupus erythematosus; SNP: Single-nucleotide polymorphism; SSc: Systemic sclerosis; Th: Helper T cell; TNF: Tumor necrosis factor

\section{Acknowledgements}

This study was supported by grants from the Japanese Society for the Promotion of Science Grants-in-Aid for Scientific Research (26713036 [to YK], 26461469 [to MT], 26461468 [to RY], and 16K19085 [to IK]), the Yokohama Foundation for Advancement of Medical Science (to YK and RY), the Naito Memorial Foundation (to YK), the Uehara Memorial Foundation (to YK), the Japan Intractable Diseases Research Foundation (to YK and MT), the Japan Rheumatism Foundation (to YK), and the Takeda Science Foundation (to RY). We express our gratitude to Momoko Ohno and Masato Kawashima for their assistance and Tom Kiper for correcting the grammar of this paper.

\section{Funding}

This study is supported by grants from the Japanese Society for the Promotion of Science Grants-in-Aid for Scientific Research (26713036 [to YK], 26461469 [to MT], 26461468 [to RY], and 16K19085 [to IK]), the Yokohama Foundation for Advancement of Medical Science (to YK and RY), the Naito Memorial Foundation (to YK), the Uehara Memorial Foundation (to YK), the Japan Intractable Diseases Research Foundation (to YK and MT), the Japan Rheumatism Foundation (to YK), and the Takeda Science Foundation (to RY).

\section{Availability of data and materials}

The datasets used and analyzed during the present study are available from the corresponding author on reasonable request.

\section{Authors' contributions}

$\mathrm{H}$ Nakano performed experiments, analyzed data and wrote the manuscript. $\mathrm{KH}, \mathrm{H}$ Nakajima, $\mathrm{YY}$, and IK performed some experiments. MT, RY, IA, and $\mathrm{H}$ Nagai coordinated the research and critically revised the manuscript. YK conceived of the experimental design, analyzed data, and wrote the manuscript. All authors read and approved the final manuscript.

\section{Ethics approval and consent to participate}

The study protocol and genetic analyses were approved by Yokohama City University Ethics Board (A140327015 and B131107009). All of the human samples were used after written informed consent was obtained.

\section{Competing interests}

MT serves as an advisor for the apremilast clinical trial for Celgene.

\section{Publisher's Note}

Springer Nature remains neutral with regard to jurisdictional claims in published maps and institutional affiliations.

\section{Author details}

${ }^{1}$ Yokohama City University Graduate School of Medicine, Department of Stem Cell and Immune Regulation, Yokohama, Japan. ${ }^{2}$ Nippon Medical School Graduate School of Medicine, Department of Allergy and Rheumatology, Tokyo, Japan. ${ }^{3}$ Yokohama City University Graduate School of Medicine, Department of Environmental Immuno-Dermatology, Yokohama, Japan. ${ }^{4}$ Yokohama City University Graduate School of Medicine, Department of Molecular Pathology, Yokohama, Japan.

Received: 13 November 2017 Accepted: 30 April 2018

Published online: 12 June 2018

\section{References}

1. Sakane T, Takeno M, Suzuki N, Inaba G. Behçet's disease. N Engl J Med. 1999:341(17):1284-91.

2. Yazici H, Fresko I, Yurdakul S. Behçet's syndrome: disease manifestations, management, and advances in treatment. Nat Clin Pract Rheumatol. 2007;3(3):148-55 
3. Ono S, Aoki K, Sugiura S, Nakayama E, Itakura K. Letter: HL-A5 and Behçet's disease. Lancet. 1973;2(7842):1383-4.

4. Remmers EF, Cosan F, Kirino Y, Ombrello MJ, Abaci N, Satorius C, et al. Genome-wide association study identifies variants in the MHC class I, IL10, and IL23R-IL12RB2 regions associated with Behçet's disease. Nat Genet. 2010:42(8):698-702.

5. Kirino Y, Bertsias G, Ishigatsubo Y, Mizuki N, Tugal-Tutkun I, Seyahi E, et al. Genome-wide association analysis identifies new susceptibility loci for Behçet's disease and epistasis between HLA-B*51 and ERAP1. Nat Genet. 2013;45(2):202-7.

6. Mizuki N, Meguro A, Ota M, Ohno S, Shiota T, Kawagoe T, et al. Genomewide association studies identify IL23R-IL12RB2 and IL10 as Behçet's disease susceptibility loci. Nat Genet. 2010;42(8):703-6.

7. Li H, Liu Q, Hou S, Du L, Zhou Q, Zhou Y, et al. TNFAIP3 gene polymorphisms confer risk for Behçet's disease in a Chinese Han population. Hum Genet. 2013;132(3):293-300

8. Xavier JM, Shahram F, Sousa I, Davatchi F, Matos M, Abdollahi BS, et al. FUT2: filling the gap between genes and environment in Behçet's disease? Ann Rheum Dis. 2015;74(3):618-24.

9. Kappen JH, Medina-Gomez C, van Hagen PM, Stolk L, Estrada K, Rivadeneira $F$, et al. Genome-wide association study in an admixed case series reveals IL12A as a new candidate in Behçet disease. PLoS One. 2015;10(3):e0119085.

10. Takeuchi M, Mizuki N, Meguro A, Ombrello MJ, Kirino Y, Satorius C, et al. Dense genotyping of immune-related loci implicates host responses to microbial exposure in Behçet's disease susceptibility. Nat Genet. 2017:49(3):438-43.

11. Kirino Y, Remmers EF. Genetic architectures of seropositive and seronegative rheumatic diseases. Nat Rev Rheumatol. 2015;11(7):401-14.

12. Aridogan BC, Yildirim M, Baysal V, Inaloz HS, Baz K, Kaya S. Serum levels of IL-4, IL-10, IL-12, IL-13 and IFN- $\gamma$ in Behçet's disease. J Dermatol. 2003;30(8):602-7.

13. Ben Ahmed M, Houman H, Miled M, Dellagi K, Louzir H. Involvement of chemokines and Th1 cytokines in the pathogenesis of mucocutaneous lesions of Behçet's disease. Arthritis Rheum. 2004;50(7):2291-5.

14. Wu Z, Zheng W, Xu J, Sun F, Chen H, Li P, et al. IL10 polymorphisms associated with Behcet's disease in Chinese Han. Hum Immunol. 2014;75(3):271-6.

15. de Wynter EA, Heyworth CM, Mukaida N, Jaworska E, Weffort-Santos A, Matushima K, et al. CCR1 chemokine receptor expression isolates erythroid from granulocyte-macrophage progenitors. J Leukoc Biol. 2001;70(3):455-60

16. Arida A, Fragiadaki K, Giavri E, Sfikakis PP. Anti-TNF agents for Behçet's disease: analysis of published data on 369 patients. Semin Arthritis Rheum. 2011;41(1):61-70.

17. Misumi M, Hagiwara E, Takeno M, Takeda Y, Inoue Y, Tsuji T, et al. Cytokine production profile in patients with Behçet's disease treated with infliximab. Cytokine. 2003;24(5):210-8.

18. Mantovani A, Marchesi F, Malesci A, Laghi L, Allavena P. Tumourassociated macrophages as treatment targets in oncology. Nat Rev Clin Oncol. 2017;14(7):399-416.

19. Verreck FA, de Boer T, Langenberg DM, Hoeve MA, Kramer M, Vaisberg E, et al. Human IL-23-producing type 1 macrophages promote but IL-10producing type 2 macrophages subvert immunity to (myco)bacteria. Proc Natl Acad Sci U S A. 2004;101(13):4560-5.

20. Guiducci C, Vicari AP, Sangaletti S, Trinchieri G, Colombo MP. Redirecting in vivo elicited tumor infiltrating macrophages and dendritic cells towards tumor rejection. Cancer Res. 2005:65(8):3437-46.

21. Endo N, Tsuboi N, Furuhashi K, Shi Y, Du Q, Abe T, et al. Urinary soluble CD163 level reflects glomerular inflammation in human lupus nephritis. Nephrol Dial Transplant. 2016;31(12):2023-33.

22. Anower AK, Shim JA, Choi B, Kwon HJ, Sohn S. The role of classical and alternative macrophages in the immunopathogenesis of herpes simplex virus-induced inflammation in a mouse model. J Dermatol Sci. 2014;73(3):198-208

23. Kirino $\mathrm{Y}$, Takeno $\mathrm{M}$, Watanabe $\mathrm{R}$, Murakami $\mathrm{S}$, Kobayashi $\mathrm{M}$, Ideguchi $\mathrm{H}$, et al. Association of reduced heme oxygenase-1 with excessive Toll-like receptor 4 expression in peripheral blood mononuclear cells in Behçet's disease. Arthritis Res Ther. 2008;10(1):R16.

24. International Study Group for Behçet's Disease. Criteria for diagnosis of Behçet's disease. Lancet. 1990;335(8697):1078-80.

25. Zhou Q, Yang D, Ombrello AK, Zavialov AV, Toro C, Zavialov AV, et al. Earlyonset stroke and vasculopathy associated with mutations in ADA2. N Engl J Med. 2014;370(10):911-20.
26. Anderson MW, Zhao S, Ai WZ, Tibshirani R, Levy R, Lossos IS, et al. C-C chemokine receptor 1 expression in human hematolymphoid neoplasia. Am J Clin Pathol. 2010;133(3):473-83.

27. Gordon IO, Freedman RS. Defective antitumor function of monocytederived macrophages from epithelial ovarian cancer patients. Clin Cancer Res. 2006;12(5):1515-24.

28. Biswas SK, Mantovani A. Macrophage plasticity and interaction with lymphocyte subsets: cancer as a paradigm. Nat Immunol. 2010;11(10):889-96.

29. Ishigaki K, Kochi Y, Suzuki A, Tsuchida Y, Tsuchiya H, Sumitomo S, et al. Polygenic burdens on cell-specific pathways underlie the risk of rheumatoid arthritis. Nat Genet. 2017:49(7):1120-5.

30. Corcione A, Tortolina G, Bonecchi R, Battilana N, Taborelli G, Malavasi F, et al. Chemotaxis of human tonsil B lymphocytes to CC chemokine receptor (CCR) 1, CCR2 and CCR4 ligands is restricted to non-germinal center cells. Int Immunol. 2002:14(8):883-92.

31. Higashi-Kuwata N, Jinnin M, Makino T, Fukushima S, Inoue Y, Muchemwa FC, et al. Characterization of monocyte/macrophage subsets in the skin and peripheral blood derived from patients with systemic sclerosis. Arthritis Res Ther. 2010;12(4):R128.

32. Franke A, Balschun T, Karlsen TH, Sventoraityte J, Nikolaus S, Mayr G, et al. Sequence variants in IL10, ARPC2 and multiple other loci contribute to ulcerative colitis susceptibility. Nat Genet. 2008:40(11):1319-23.

33. Glocker EO, Kotlarz D, Boztug K, Gertz EM, Schaffer AA, Noyan F, et al. Inflammatory bowel disease and mutations affecting the interleukin-10 receptor. N Engl J Med. 2009;361(21):2033-45.

34. Hou S, Xiao X, Li F, Jiang Z, Kijlstra A, Yang P. Two-stage association study in Chinese Han identifies two independent associations in CCR1/ CCR3 locus as candidate for Behçet's disease susceptibility. Hum Genet. 2012;131(12):1841-50.

35. Takeuchi M, Kastner DL, Remmers EF. The immunogenetics of Behçet's disease: a comprehensive review. J Autoimmun. 2015;64:137-48.

36. Lee TS, Chau LY. Heme oxygenase-1 mediates the anti-inflammatory effect of interleukin-10 in mice. Nat Med. 2002;8(3):240-6.

37. Naito Y, Takagi T, Higashimura Y. Heme oxygenase-1 and anti-inflammatory M2 macrophages. Arch Biochem Biophys. 2014:564:83-8.

38. Kirino Y, Zhou Q, Ishigatsubo Y, Mizuki N, Tugal-Tutkun I, Seyahi E, et al. Targeted resequencing implicates the familial Mediterranean fever gene MEFV and the toll-like receptor 4 gene TLR4 in Behçet disease. Proc Natl Acad Sci U S A. 2013;110(20):8134-9.

39. Han KH, Gonzalez-Quintial R, Peng Y, Baccala R, Theofilopoulos AN, Lerner RA. An agonist antibody that blocks autoimmunity by inducing antiinflammatory macrophages. FASEB J. 2016;30(2):738-47.

40. Li F, Yang Y, Zhu X, Huang L, Xu J. Macrophage polarization modulates development of systemic lupus erythematosus. Cell Physiol Biochem. 2015;37(4):1279-88.

41. Kirino Y, Takeno M, Murakami S, Kobayashi M, Kobayashi H, Miura K, et al. Tumor necrosis factor a acceleration of inflammatory responses by downregulating heme oxygenase 1 in human peripheral monocytes. Arthritis Rheum. 2007;56(2):464-75.

42. Hatemi G, Melikoglu M, Tunc R, Korkmaz C, Turgut Ozturk B, Mat C, et al. Apremilast for Behçet's syndrome-a phase 2, placebo-controlled study. N Engl J Med. 2015;372(16):1510-8.

43. Zeller T, Wild P, Szymczak S, Rotival M, Schillert A, Castagne R, et al. Genetics and beyond - the transcriptome of human monocytes and disease susceptibility. PLoS One. 2010;5(5):e10693.

44. Hughes T, Ture-Ozdemir F, Alibaz-Oner F, Coit P, Direskeneli H, Sawalha AH. Epigenome-wide scan identifies a treatment-responsive pattern of altered DNA methylation among cytoskeletal remodeling genes in monocytes and CD4+ T cells from patients with Behcet's disease, Arthritis Rheumatol. 2014; 66(6):1648-1658.

45. Hilhorst M, Shirai T, Berry G, Goronzy JJ, Weyand CM. T cellmacrophage interactions and granuloma formation in vasculitis. Front Immunol. 2014;5:432

46. Hirohata S, Kikuchi H. Behçet's disease. Arthritis Res Ther. 2003;5(3):139-46. 